\title{
3D printing technology applied to orthosis manufacturing: narrative review
}

\author{
Yoo Jin Choo ${ }^{1}$, Mathieu Boudier-Revéret ${ }^{2}$, Min Cheol Chang ${ }^{3}$ \\ ${ }^{1}$ Production R\&D Division Advanced Interdisciplinary Team, Medical Device Development Center, Daegu-Gyeongbuk Medical Innovation, Daegu, \\ Korea; ${ }^{2}$ Department of Physical Medicine and Rehabilitation, Centre Hospitalier de l'Université de Montréal, Montreal, Canada; ${ }^{3}$ Department of \\ Rehabilitation Medicine, College of Medicine, Yeungnam University, Daegu, Korea \\ Contributions: (I) Conception and design: YJ Choo, MC Chang; (II) Administrative support: None; (III) Provision of study materials or patients: \\ None; (IV) Collection and assembly of data: YJ Choo; (V) Data analysis and interpretation: YJ Choo, MC Chang; (VI) Manuscript writing: All \\ authors; (VII) Final approval of manuscript: All authors. \\ Correspondence to: Min Cheol Chang, MD. Department of Physical Medicine and Rehabilitation, College of Medicine, Yeungnam University, 317-1, \\ Daemyungdong, Namku, Taegu, 705-717, Korea. Email: wheel633@ynu.ac.kr.
}

\begin{abstract}
Recently, three-dimensional (3D) printing technology has gradually been applied to the field of orthoses. This narrative review aimed to investigate the effect of $3 \mathrm{D}$ printed orthoses compared to conventional orthoses (non-3D printed orthoses). We searched MEDLINE for articles published up to July 27, 2020, and the main search phrases for identifying related articles were "3D printed orthosis", “3D printed orthoses", "3D printed braces”, “3D printed splints”, "3D printing orthosis", "3D printed orthoses", "3D printing braces" and "3D printing splints". We included articles that applied 3D printed orthoses to patients or healthy participants and excluded those not written in English, conference abstracts or presentations, and reviews. A total of 237 papers were identified, and qualifications were evaluated based on the title, abstract, and full text. A total of 22 articles were finally included in the analysis. The 3D printed orthoses showed similar or superior effects on biomechanical parameters and kinematic parameters such as wrist-hand function, wrist spasticity, arch height index, foot plantar pressure, and joint range of motion (ROM). In addition, 3D printed orthoses had high satisfaction and comfort compared to conventional orthoses. We believe that 3D printed orthoses can replace conventional ones, and they are expected to gain more popularity in the future.
\end{abstract}

Koywords: 3D printing technology; rehabilitation technology; orthosis; 3D printed orthosis

Submitted May 28, 2020. Accepted for publication Sep 10, 2020.

doi: 10.21037/apm-20-1185

View this article at: http://dx.doi.org/10.21037/apm-20-1185

\section{Introduction}

An orthosis is a device used to assist the functions of the human body. They usually have the following purposes: body protection, restricting motion, weight-bearing assistance, movement assistance, and deformity prevention or correction. Thus, orthoses are widely applied to patients with physical dysfunction and disability due to muscular dysfunctions, such as fractures, sprains, arthropathy and tendinopathy, and neurological disorders in the brain, spinal cord, and peripheral nerves $(1,2)$.
The conventional method for manufacturing orthoses is relatively time-consuming. Further, it is necessary to manually correct the shape and dimensions of the orthosis according to the patient's body. In addition, it is challenging to produce multiple customized orthoses with the same quality, and complex designs sometimes can be implemented. However, using recently developed three-dimensional (3D) printing technology, orthoses can be designed with exact dimensions through a computer graphic program, and the aforementioned disadvantages of the conventional method can be sufficiently addressed 


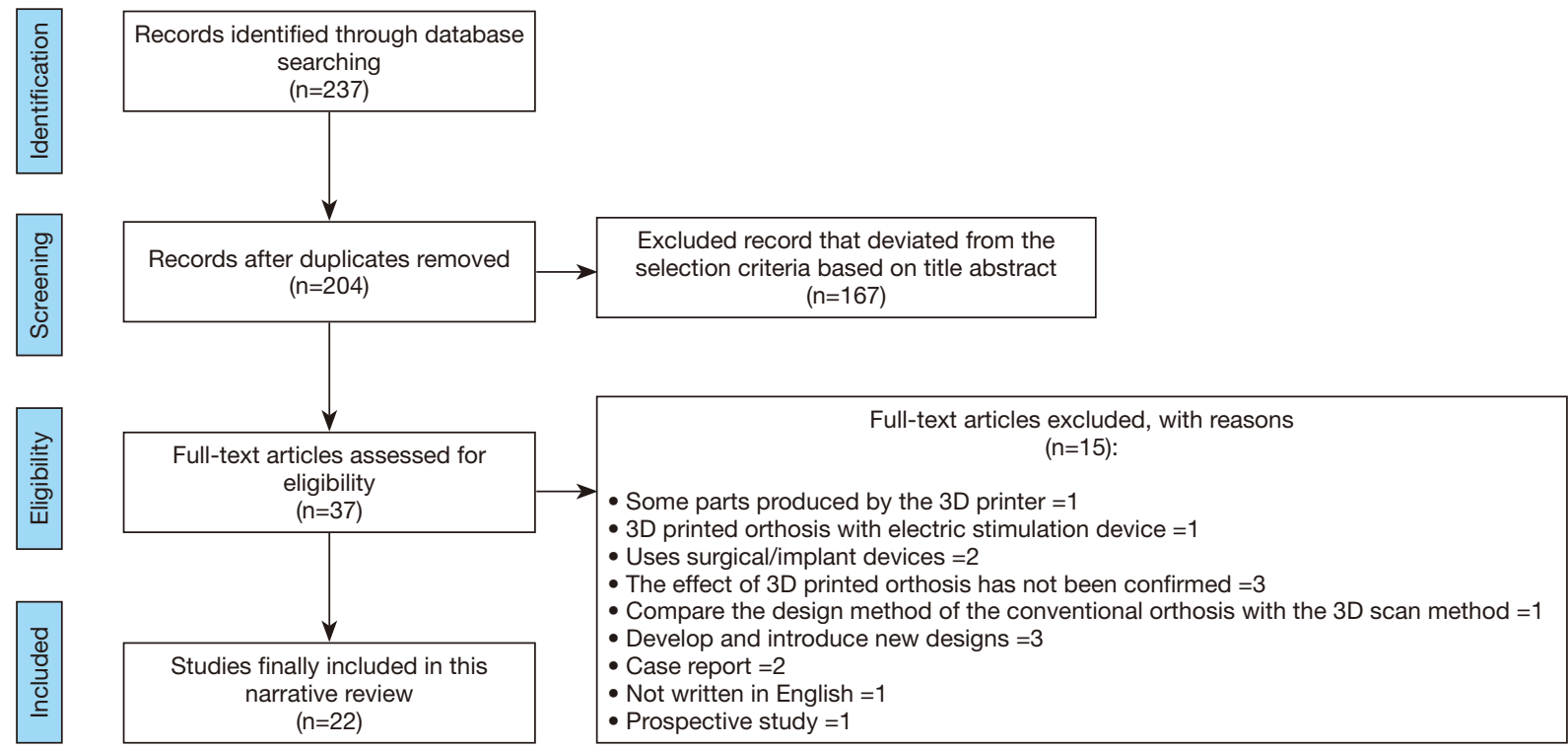

Figure 1 Flow chart showing the search results for the study.

because $3 \mathrm{D}$ printers are highly accurate. Thus, using $3 \mathrm{D}$ printing technology, it is possible to design an orthosis with accurate numerical values of the dimensions through a design program and create structures that are difficult to implement manually (3-5). Additionally, while a manually produced orthosis requires approximately 1 week to produce, a $3 \mathrm{D}$ printer can perform this task in a day $(6,7)$. Therefore, in the field of orthoses, significant attention is being paid to orthoses manufactured with $3 \mathrm{D}$ printing technologies.

In this study, we investigate the recent advancements in $3 \mathrm{D}$ printed orthoses by reviewing prior studies. We present this article in accordance with the narrative review reporting checklist (available at http://dx.doi.org/10.21037/ apm-20-1185).

\section{Methods}

We set "3D printed orthosis", "3D printed orthoses", "3D printed braces", "3D printed splints", "3D printing orthosis", "3D printed orthoses", "3D printing braces" and "3D printing splints" as keywords in PubMed, CINAHL Complete \& MEDLINE Complete, Cochrane, Embase, and Scopus to search for articles published up to July 27, 2020. The criteria for article inclusion are as follows: (I) 3D printed orthosis applied to a patient or a healthy subject, (II) area of application should be an upper limb, lower limb, or spine, (III) prospective study, and (IV) the availability of a full-text. The criteria for article exclusion are as follows: (I) object of comparison is a surgical treatment, or a conservative treatment other than orthosis, (II) orthosis manufactured by adding electrical stimulation device or robotic device, (III) replacement product for human body structure made for surgery or implant, (IV) some parts produced by a 3D printer, and (V) case reports, conference abstracts or presentations, review articles, and non-English publications.

The level of evidence was defined using the Research Pyramid model. The evaluation criteria are as follows: (I) level 1: systematic reviews and meta-analysis of randomized controlled trials (RCTs), (II) level 2: one or more RCTs, (III) level 3: controlled trials without randomization (at least two groups), (IV) level 4: case-control or cohort study, (V) level 5: systematic review of descriptive and qualitative study, (VI) level 6: single descriptive or qualitative study, and (VII) level 7: expert opinion. The results of the level of evidence evaluation of the papers included in this review were level 2 in six papers (6-11), level 3 in six papers $(4,5,12-15)$, and level 4 in ten papers $(3,16-24)$.

\section{Results}

We identified 237 relevant articles. Among them, a total of 22 were selected (3-24), excluding articles that did not match the inclusion criteria (Figure 1). Of the 22 
studies, there were 8 studies related to the upper limbs $(6,11,16-19,21,24), 13$ related to the lower limbs (3-5,7-10,12,14,15,20,22,23), and 1 related to the spine (13). The characteristics of each study are summarized in Table 1. The following subsection explains how orthoses are made for each study.

\section{Upper limb orthosis}

Of the eight articles, two were randomized trials $(6,11)$, and six were prospective observational studies (16-19,21,24).

In 2018, Kim et al. (6) manufactured a $3 \mathrm{D}$ printed orthosis in which the wrist joint was slightly dorsiflexed and free finger movements were possible, and the length was to the center of the forearm. The control group used ready-made cock-up splint. In 2020, Zheng et al. (11) manufactured the $3 \mathrm{D}$ printed orthosis in shape of a functional hand position $\left(10^{\circ}-15^{\circ}\right.$ extension of the wrist, $40^{\circ}-45^{\circ}$ flexion of the metacarpophalangeal (MP) and proximal interphalangeal, and thumb abduction). The control group used a low-temperature thermoplastic plate orthosis. In 2017, Chen et al. (16) 3D printed an orthosis designed to wrap around from the center of mid-forearm to the MP except for the thumb. In addition, the forearm part had several small holes for ventilation and wearerfriendliness, and the shells surrounding the upper and lower parts of the forearm-wrist-hand were sectioned and could be fixed using a Velcro strap. The 3D printed orthosis used by Portnova et al. (21) in 2018 was a wrist-driven orthosis, and each component of the orthosis was manufactured in various sizes to suit different users. The orthosis was designed with the feedback from three users and six orthotists. In 2018, Wang et al. (24) 3D printed the orthosis in the form of a fingerboard that straightens five fingers and maintains an abduction position. Small holes were drilled into the palmar side to make it breathable and comfortable for the user when wearing the orthosis. Only the base plate was produced using a 3D printer, and a Velcro strap was used for fixing it to the hand and finger. The 3D printed orthosis used by Guida et al. (18) had a design in which the wrists and hands were almost horizontally aligned, and the thumb was fixed at a slight abduction. The length of the body ranged from approximately proximal twothird of the forearm to a little above the MP. The shell was made in a double-shell design, and a hole was created on the entire shell for ventilation and lightness. In 2019, Lee et al. (19) 3D printed an orthosis designed to support from approximately the mid-forearm area to the MP and wrap only the MP area. The back of the hand and forearm were fixed with Velcro straps. As the patient wanted to perform daily life activities such as typing, eating, and writing. The hypothenar eminence area has a connector to fix a typing device or spoon, and a small ring was included between the thumb and index finger of the orthosis such that a pencil could be used. Furthermore, several small holes were made for breathability. In 2020, Chu et al. (17) measured the hands of 120 subjects and created a $3 \mathrm{D}$ parametric hand model. When manufacturing the orthosis, an offset of 1-2 $\mathrm{mm}$ was applied to the interphalangeal joint of the thumb and MP, and another offset of 2-3 mm was applied between the fifth metacarpal bone and the carpometacarpal joint. Thus, a suitable space was created on the inner surface between the thumb and the orthosis. This orthosis was designed to wrap under the MP, back of the hand, under the interphalangeal of thumb, and palm. There were no holes for ventilation, and because it consisted of one shell, a separate strap was not required.

\section{Lower limb orthosis}

Of the 13 related articles, there were four randomized trials $(7-10)$ and 10 prospective observational studies $(3-5,12,14,15,20,22,23)$.

In 2017, Telfer et al. (7) designed a 3D printed insole using foam-box foot impressions that were $3 \mathrm{D}$ scanned. The metatarsal area of the insole was layered, and the pressure was distributed when a vertical load was applied. The shape of the insole was similar to that of a ready-made insole. In 2019, Mo et al. (8) produced a 3D printed foot orthosis, with the subtalar joint positioned neutrally. A 3-mm PORON cover was added to the foot orthosis. Two studies were conducted in 2019 by $\mathrm{Xu}$ et al. $(9,10)$. One of them was targeted at patients with plantar fasciitis (9). The pressure distribution was eased using lateral wedges in the heel of the insole and metatarsal pad. In a study involving patients with bilateral flat foot (10), a customized $3 \mathrm{D}$ printed insole was constructed that firmly supported the medial arch of the foot such that it did not collapse. In this $3 \mathrm{D}$ printed insole, the part surrounding the heel was deeper than in ready-made or normally customized insole, which increased the contact area where the foot touches the insole, thereby distributing the pressure. The 3D printed foot orthosis produced in two studies conducted by Telfer et al. in $2013(22,23)$ were semi-rigid devices and threefourth of the total foot length. The 3D printed orthosis used by Dombroski et al. (4) was designed through a $3 \mathrm{D}$ 
Table 1 Characteristics of included studies

\begin{tabular}{|c|c|c|c|c|c|c|c|c|c|}
\hline No. & Study & Study design & Participants & $\begin{array}{l}\text { Applied body } \\
\text { parts }\end{array}$ & Applied orthoses & $\begin{array}{l}\text { Digital fabrication } \\
\text { pathway }\end{array}$ & Type of $3 D$ printer & Material of 3DP & Result \\
\hline 1 & $\begin{array}{l}\text { Kim et al. } \\
\text { (6) }\end{array}$ & $\begin{array}{l}\text { Randomized } \\
\text { trial }\end{array}$ & $\begin{array}{l}\mathrm{N}=22(\mathrm{E}: 11, \mathrm{C}: 11) \\
\text { overuse syndrome in } \\
\text { the upper wrist area }\end{array}$ & $\begin{array}{l}\text { Upper limbs } \\
\text { (wrist-hand) }\end{array}$ & $\begin{array}{l}\text { 3D printed wrist orthosis (3DP), } \\
\text { cock-up splint (CO) }\end{array}$ & 3D scanner & $\begin{array}{l}\text { Fused filament fabrication [FINEBOT } \\
\text { Z420 3D printer (TPC Mechatronics } \\
\text { Inc., Incheon, Korea)] }\end{array}$ & $\begin{array}{l}\text { Thermoplastic } \\
\text { polyurethane }\end{array}$ & 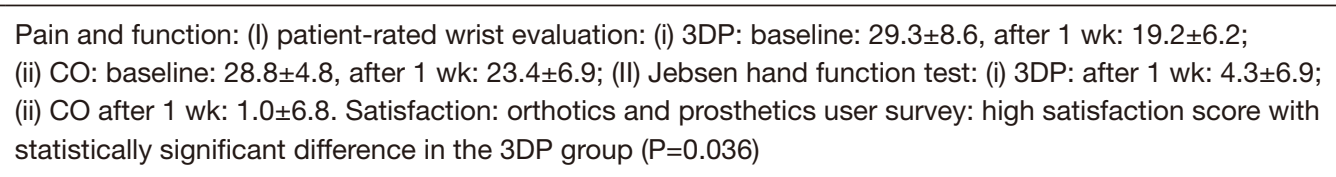 \\
\hline 2 & $\begin{array}{l}\text { Zheng et al. } \\
\text { (11) }\end{array}$ & $\begin{array}{l}\text { Randomized } \\
\text { trial }\end{array}$ & $\begin{array}{l}N=60 \text { (E: } 30, C: 30) \\
\text { Colles' fractures }\end{array}$ & $\begin{array}{l}\text { Upper limbs } \\
\text { (wrist-hand) }\end{array}$ & $\begin{array}{l}\text { 3D printed wrist-hand orthosis } \\
\text { (3DP), manually produced } \\
\text { small splint (low-temperature } \\
\text { thermoplastic plate orthosis, CO) }\end{array}$ & CAD & Nonexistent information & Light-activated resin & $\begin{array}{l}\text { Spasticity/stability, mobility, hand function/pain/swelling: modified Ashworth scale (wrist flexor)/Fugl-Meyer } \\
\text { assessment score/visual analog scale/swelling score: (I) 3DP: baseline: mean 2.2/3.9/3.0/3.0, after } 6 \text { wk: } \\
\text { mean 1.6/5.2/2.5/1.0; (II) CO: baseline: mean 2.2/3.9/3.0/2.5, after } 6 \text { wk: mean 2.0/4.1/3.0/2.0 }\end{array}$ \\
\hline 3 & $\begin{array}{l}\text { Chen et al. } \\
\text { (16) }\end{array}$ & $\begin{array}{l}\text { Prospective } \\
\text { observational } \\
\text { study }\end{array}$ & $\begin{array}{l}\mathrm{N}=10 \text {, distal radius } \\
\text { fracture }\end{array}$ & $\begin{array}{l}\text { Upper limbs } \\
\text { (wrist-hand) }\end{array}$ & 3D printed cast & $\begin{array}{l}\text { Magnetic resonance } \\
\text { imaging and } \\
\text { computerised } \\
\text { tomography }\end{array}$ & $\begin{array}{l}\text { Selective laser sintering (EOS P395, } \\
\text { Germany)/stereolithography printer } \\
\text { (RS4500, UnionTech, China) }\end{array}$ & $\begin{array}{l}\text { Polypropylene and } \\
\text { polyamide }\end{array}$ & $\begin{array}{l}\text { Clinical efficacy (stability, blood circulation, pain, pressure): } 9.8 \text { out of } 12 \text { points. Satisfaction: } 11.5 \text { out of } \\
15 \text { points }\end{array}$ \\
\hline 4 & $\begin{array}{l}\text { Portnova et } \\
\text { al. (21) }\end{array}$ & $\begin{array}{l}\text { Prospective } \\
\text { observational } \\
\text { study }\end{array}$ & $\begin{array}{l}\mathrm{N}=3 \text {, spinal cord } \\
\text { injury }\end{array}$ & $\begin{array}{l}\text { Upper limbs } \\
\text { (wrist-hand) }\end{array}$ & 3D printed wrist-driven orthosis & CAD & $\begin{array}{l}\text { Fused filament fabrication } \\
\text { (FlashForge Creator Pro, FlashForge, } \\
\text { Rowland Heights, CA, USA) }\end{array}$ & $\begin{array}{l}\text { Polylactic acid } \\
\text { plastic }\end{array}$ & $\begin{array}{l}\text { Average score of function/aesthetics/comfort from user: 6.8/7.7/7.7. Jebsen hand function test: (I) patient } \\
\text { 1: improvement in cards, small objects, checkers, and empty cans sections; (II) patient 2: improvement in } \\
\text { writing, eating, checkers, empty cans, and full cans sections; (III) patient 3: improvement in writing, small } \\
\text { objects, and checkers sections }\end{array}$ \\
\hline 5 & $\begin{array}{l}\text { Wang et al. } \\
\text { (24) }\end{array}$ & $\begin{array}{l}\text { Prospective } \\
\text { observational } \\
\text { study }\end{array}$ & $\mathrm{N}=18$, stroke & $\begin{array}{l}\text { Upper limbs } \\
\text { (hand) }\end{array}$ & 3D printing fingerboard & 3D scanner & Fused deposition modeling & Polylactic acid & $\begin{array}{l}\text { Pain: visual analog scale: before: } 2.0 \pm 1.58 \text {, after } 3 \text { wk: } 1.85 \pm 1.34 \text {, after } 3 \mathrm{mo:}: 1.69 \pm 1.03 \text {. Hand grip } \\
\text { strength: hand grip dynamometer: before: } 0.018 \pm 0.041 \text {, after } 3 \text { wk: } 0.025 \pm 0.054 \text {, after } 3 \text { mo: } 0.032 \pm 0.067 \text {. } \\
\text { ROM of hand joints: (I) wrist flexion (active): before: } 2.62 \pm 8.27 \text {, after } 3 \text { wk: } 5.08 \pm 11.6 \text {, after } 3 \text { mo: } 5.83 \pm 13.8 \text {; } \\
\text { (II) wrist extension (active): before: } 1.0 \pm 2.77 \text {, after } 3 \text { wk: } 3.62 \pm 9.83 \text {, after } 3 \mathrm{mo:} 3.85 \pm 11.2 \text {. Muscle tension } \\
\text { of the hand: modified Ashworth scale: before: } 2.38 \pm 0.65 \text {, after } 3 \text { wk: } 2.31 \pm 0.48 \text {, after } 3 \text { mo: } 1.7 \pm \pm 0.70 \text {. } \\
\text { Hand movement function: Brunnstrom approach: before: } 2.77 \pm 0.83 \text {, after } 3 \text { wk: } 3.00 \pm 0.82 \text {, after } 3 \mathrm{mo:} \\
3.23 \pm 0.83\end{array}$ \\
\hline 7 & $\begin{array}{l}\text { Lee et al. } \\
(19)\end{array}$ & $\begin{array}{l}\text { Prospective } \\
\text { observational } \\
\text { study }\end{array}$ & $\mathrm{N}=1$, stroke & $\begin{array}{l}\text { Upper limbs } \\
\text { (wrist-hand) }\end{array}$ & $\begin{array}{l}\text { 3D printed wrist-hand orthosis } \\
\text { (3DP), ready-made assistive } \\
\text { device (CO) }\end{array}$ & 3D scanner & $\begin{array}{l}\text { Fused filament fabrication [FB9600 } \\
\text { printer (TPC Mechatronics Inc., } \\
\text { Incheon, Korea)] }\end{array}$ & $\begin{array}{l}\text { Thermoplastic } \\
\text { polyurethane (3DP), } \\
\text { polylactic acid } \\
\text { (connector) }\end{array}$ & $\begin{array}{l}\text { Jebsen hand function test (writing/feeding/typing) (s): 3DP: 43.2/12.3/22.3; CO: 42.6/25.1/24.2. Quebec } \\
\text { user assessment (writing/feeding/typing) (s): 3DP: 31/35/38; CO: 30/28/30 }\end{array}$ \\
\hline 8 & $\begin{array}{l}\text { Chu et al. } \\
(17)\end{array}$ & $\begin{array}{l}\text { Prospective } \\
\text { observational } \\
\text { study }\end{array}$ & $\mathrm{N}=1$, healthy & $\begin{array}{l}\text { Upper limbs } \\
\text { (hand) }\end{array}$ & 3D hand orthosis & 3D scanner & $\begin{array}{l}\text { Fused deposition modeling (Grandore } \\
\text { Fika 2: Stratasys, Eden Prairie, MN, } \\
\text { USA) }\end{array}$ & $\begin{array}{l}\text { Thermoplastic } \\
\text { elastomer }\end{array}$ & $\begin{array}{l}\text { Usability test: possible to unscrew a bottle cap, handwrite, play computer game with a mouse, and text on } \\
\text { a smartphone. Quebec user evaluation of satisfaction with assistive technology: mean satisfaction level: } 4.9\end{array}$ \\
\hline 9 & $\begin{array}{l}\text { Telfer et al. } \\
\text { (7) }\end{array}$ & $\begin{array}{l}\text { Randomized } \\
\text { trial }\end{array}$ & $\begin{array}{l}\mathrm{N}=20(\mathrm{E}: 20, \mathrm{C}: 20) \\
\text { diabetes }\end{array}$ & $\begin{array}{l}\text { Lower limbs } \\
\text { (foot) }\end{array}$ & $\begin{array}{l}3 \mathrm{D} \text { printed insole, standard } \\
\text { insole }\end{array}$ & 3D scanner & $\begin{array}{l}\text { Fused deposition modeling [Airwolf } \\
\text { HD2x printer (Airwolf 3D, Costa Mesa, } \\
\text { CA, USA)] }\end{array}$ & Polyethylene acetate & $\begin{array}{l}\text { Peak pressure result (kPa): (I) 3D printed insole: approximately 285; (II) standard shaped insole: } \\
\text { approximately } 245\end{array}$ \\
\hline 10 & Mo et al. (8) & $\begin{array}{l}\text { Randomized } \\
\text { trial }\end{array}$ & $\begin{array}{l}\mathrm{N}=13(\mathrm{E}: 13, \mathrm{C}: \\
\text { 13), asymptomatic } \\
\text { runners with } \\
\text { excessive foot } \\
\text { pronation }\end{array}$ & $\begin{array}{l}\text { Lower limbs } \\
\text { (foot) }\end{array}$ & $\begin{array}{l}\text { 3D printed foot orthosis (3DP), } \\
\text { traditional plaster molded (TPM) }\end{array}$ & 3D scanner & Nonexistent information & $\begin{array}{l}\text { Nylon (Polymarker } \\
\text { PolyMide' }\end{array}$ & 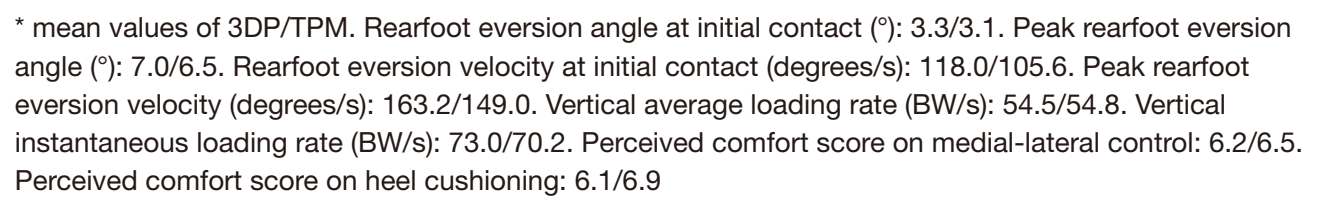 \\
\hline 11 & Xu et al. (9) & $\begin{array}{l}\text { Randomized } \\
\text { trial }\end{array}$ & $\begin{array}{l}N=60 \text { (E: } 30, C: 30) \\
\text { plantar fasciitis }\end{array}$ & $\begin{array}{l}\text { Lower limbs } \\
\text { (foot) }\end{array}$ & $\begin{array}{l}\text { 3D printed insole (3DP), } \\
\text { prefabricated insole }\end{array}$ & CAD & $\begin{array}{l}\text { Fused deposition modelling } \\
\text { (Bodyarch } \mathrm{X} \text { printer }{ }^{\text {) }}\end{array}$ & Polyethylene acetate & $\begin{array}{l}\text { Pain: visual analog scale: (I) 3DP: baseline: } 7.34 \pm 3.43 \text {, after } 8 \mathrm{wk}: 3.12 \pm 0.51 \text {; (II) prefabricated insole: } \\
\text { baseline: } 8.72 \pm 3.93 \text {, after } 8 \text { wk: } 5.25 \pm 1.22\end{array}$ \\
\hline 12 & $\begin{array}{l}\text { Xu et al. } \\
\text { (10) }\end{array}$ & $\begin{array}{l}\text { Randomized } \\
\text { trial }\end{array}$ & $\begin{array}{l}N=80 \text { (E: } 40, C: 40) \\
\text { bilateral flatfoot }\end{array}$ & $\begin{array}{l}\text { Lower limbs } \\
\text { (foot) }\end{array}$ & $\begin{array}{l}3 \mathrm{D} \text { printed insole (3DP), } \\
\text { prefabricated insole }\end{array}$ & CAD & $\begin{array}{l}\text { Fused deposition modelling } \\
\text { (Bodyarch X1 printer })^{\text {) }}\end{array}$ & Polyethylene acetate & $\begin{array}{l}\text { Pain: visual analog scale: (I) 3DP: baseline: } 7.91 \pm 3.11 \text {, after } 8 \text { wk: } 2.42 \pm 0.13 \text {; (II) prefabricated insole: } \\
\text { baseline: } 8.12 \pm 1.21 \text {, after } 8 \text { wk: } 5.92 \pm 1.19\end{array}$ \\
\hline
\end{tabular}

Table 1 (continued) 
Table 1 (continued)

No. Study

Study design

Participants

Applied bod

Applied orthoses

Digital fabrication

Type of 3D printer

Material of 3DP

Result

Lower limbs 3D printed foot orthosis $3 \mathrm{D}$ scanner

Fused deposition modeling [3D

\section{Significan}

$\begin{array}{llll}\text { Telfer et al. } & \text { Prospective } & \mathrm{N}=24 \text { (E: 12, C: 12), } & \text { Lo } \\ \text { (22) } & \text { observational } & \text { pronated foot } & \text { (foot) }\end{array}$

printing system (RapMan; Bits from

Polyactide study

14 Telfer et al. Prospective $\mathrm{N}=24$ (E: 12, C: 12), Lower limbs 3D printed foot orthosis

3D scanner

Fused deposition modeling [3D

Polyactide printing system (RapMan; Bits from Bytes, Clevedon, UK)] study

15 Dombroski Prospective observa
study

$\mathrm{N}=1$, healthy (foot)

Fused deposition modeling [desktop 3D printer (Makerbot ${ }^{\oplus}$, Makerbot Industries, Brookiyn, NY, USA]] butadiene styren Fused depositon modeling fdestop filament $3 \mathrm{D}$ printing system (3D Touch; Bits from Bytes, Clevedon, UK)]

thermoplastic

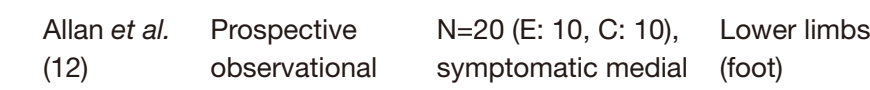
condition

study knee osteoarthritis

Eight types of 3D printed insole 3D scanner with lateral wedging-types: $3 / 4$ rearfoot (B), $10^{\circ}$ rearfoot and $5^{\circ}$ forefoot (C), and $10^{\circ}$ rearfoot (D), respectively

17 Cha et al. Prospective

Lower limbs

3 p printed ankle-foot orthosis (3DP), conventional Ankle-foo

3D scanner orthosis

18 Jin et al. (5) Prospective $\mathrm{N}=30$, health Lower limbs

3D printed insole with arch lift (A), 3D printed insole (B),

standardized pre-made insole (C)

3D scanner

observationaly
study

19 Liu et al. Prospective $\mathrm{N}=12$, stroke

Lower limbs 3D printed ankle-foot orthosis 3D scanner observationa

(ankle-foot)

(3DP)

20 Mannisi Prospective $\mathrm{N}=7$, knee

et al. (14) observational osteoarthritis

study

21 Tarrade Prospective

et al. (15) observation

$\mathrm{N}=34$, standing Lower limbs $3 \mathrm{D}$ printed custom-made foot $3 \mathrm{D}$ scanner

Three types of 3D printed insole 3D scanner with $0^{\circ}, 5^{\circ}$, and $10^{\circ}$ of latera (n)
Fused filament fabrication $\left(\mathrm{FB} 9600^{\oplus}, \quad\right.$ Thermoplastic TPC Mechatronics Corp., Incheon, polyurethane Korea)

\section{Fused deposition modelling}

(Bodyarch X1 printer ${ }^{\mathbb{9}}$ )

Polyethylene

Multi Jet Fusion

(Jet Fusion 3D 4200, HP, USA)

3D high reusability

polyamide 12

Fused deposition modelling (desktop Soft thermoplastic (DD

Laser sintering

Polyamide 12

Biodegradable

polylactide Airwolf $3 \mathrm{D}$ printers, Californi USA

peak/mean lateral forefoot: $P<0.001$

Significant linear effect in kinematic and kinetic variables: peak/mean rearfoot eversion: $P<0.001$; mean internal tibial rotation: $P=0.003$; peak/mean ankle eversion moment: $P<0.001$; second peak knee

adduction $\mathrm{m}$

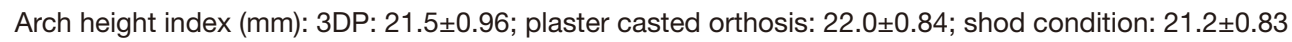

Percentage changes in biomechanical variables: (I) first peak knee adduction moment: significant main effects in length and wedging condition except A: $P=0.038$ and $P<0.0001$. (II) second peak knee adduction moment: significant main effects in length and wedging condition: $\mathrm{P}=0.018$ and $\mathrm{P}<0.0001$ (III) knee adduction moment impulse: significant main effects in length and wedging condition: $\mathrm{P}=0.022$ and $\mathrm{P}<0.0001$

Gait speed (cm/s): 3DP/conventional AFO/no AFO: 56.5/56.5/42.2. Stride length (cm): 3DP/conventional AFO/no AFO: 70.9/70.9/63.2. Step width (cm): 3DP/conventional AFO/no AFO: 15.9/17.1/17.9. Single stance ratio between the right/left sides (\%): 3DP/conventional AFO/no AFO: 80.4/79.7/69.7. Quebec user evaluation of satisfaction with assistive technology $3 \mathrm{DP} /$ conventional AFO: 4.5/4.1

Peak pressure in rearfoot (kPa): A/B/C: approximately 170/200/260. Force-time integral in forefoot (\%): A/B/C: approximately 22/25/35. Range of medial-lateral center of pressure (mm): forefoot contact phase lateral center of pressure (cm/s): forefoot contact phase (A/B/C): approximately 15/16/19

Temporal-spatial parameters (before/after using 3DP): velocity ( $\mathrm{m} / \mathrm{s})$ : $0.17 \pm 0.06 / 0.20 \pm 0.07$; cadence (times/min): $47.0 \pm 14.4 / 53.8 \pm 15.5$; stride length $(\mathrm{m}): 0.43 \pm 0.11 / 0.48 \pm 0.11$; gait cycle (s): $2.8 \pm 1.1 / 2.5 \pm 1.0$ double limb support phase (\%): $36.3 \pm 5.6 / 33.6 \pm 5.2$; step length difference $(\mathrm{m})$ : $0.16 \pm 0.12 / 0.10 \pm 0.09$

Medial to lateral ratio for the impulse of the compressive force: shoe only: $0 \% 15^{\circ} / 10^{\circ}$; mean 2.68/2 93/2 80/2.75. Walking speed (m/s): shoe only: $0^{\circ} / 5^{\circ} / 10^{\circ}$. mean: $1.15 / 1.15 / 1.18 / 1.17$

Numerical rating scale (pain/comfortheavy legs): before 3DP: $5.3 \pm 2.4 / 5.9 \pm 2.8 / 5.3 \pm 3.1$, after 3DP: 2.0 $\pm 2.0 / 1.4 \pm 2.1 / 2.1 \pm 2.4$. Foot health status questionnaire (pain//unction/footwear/general foot heal $6 / 5900250$. Center of pressure parameters (with/without 3DP): total displacement of sway (mm)

$1,370.6 \pm 380.6 / 1,682.9 \pm 937.6$; amplitude of anteroposterior displacement $(\mathrm{mm}): 30.8+17 / 40.4 \pm 26.5$ amplitude of medial-lateral displacement $(\mathrm{mm}): 20.1 \pm 9.7 / 30.4 \pm 15.9$; mean anteroposterior velocity $(\mathrm{mm} / \mathrm{s})$ : $17.7 \pm 5.7 / 19.8 \pm 15.3 ;$ mean medial-lateral velocity $(\mathrm{mm} / \mathrm{s}): 16.7 \pm 4.5 / 21.9 \pm 11.2$; sway area $(95 \%$ of COP position) $\left(\mathrm{mm}^{2}\right)$ : $397.5 \pm 755.2 / 462.9 \pm 659.8$

22 Kuo et al. Prospective $\mathrm{N}=41$, healthy Spine 3D printed customized collar

(3DP), Aspen Vista collar,
Sport-aid cervical collar

Head/neck/trunk angle [mean value ( $)$ ]: (1) 3DP collar: sitting with back support: 12.25/5.31/0.03, sitting without back support: 24.36/18.74/14.49, standing: 13.9/3.26/11.73; (II) Aspen Vista: sitting with back support: 13.81/6.85/1.07, sitting without back support: 29.57/22.74/15.87, standing: 15.24/3.51/11.25; (III) sport-aid: sitting with back support: 20.49/7.29/0.2, sitting without back support: 32.94/21.53/14.52, standing: $21.41 / 3.84 / 10.88$

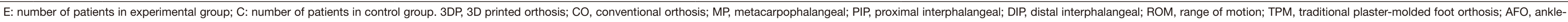
foot orthosis; $\mathrm{COP}$ center of pressure: TMO, traditional mold-based orthosis. 
scan. Although the shape was not specifically mentioned, it had a feature that resulted in a higher arch height index than the control group. Allan et al. (12) evaluated the effect using eight types of 3D printed insole, including three-fourth of the length or full length with (I) $0^{\circ}$ rearfoot, (II) $5^{\circ}$ rearfoot, (III) $10^{\circ}$ rearfoot and $5^{\circ}$ forefoot, and (IV) $10^{\circ}$ rearfoot. All individuals' foot were scanned, and the insoles were made according to the shape of each foot. The orthosis used by Cha et al. (3) was an open type of heel and malleolus. Similar to the conventional orthosis, the front side was open with a shape that wrapped more to the front of the lower limb. The calf shell and plantar foot-plate length were approximately half of the conventional orthosis. In 2019, Jin et al. (5) produced the basic 3D printed insole in the form of a raised heel structure and an arched arch lift. The experimental group was divided into two groups; Group A had modified insoles such that the lateral-longitudinal portion was completely in contact with the bottom. The insoles of Group B were not applied with an arch lift. Liu et al. (20) produced 3D printed ankle-foot orthosis similar to posterior leaf spring orthosis. The ankle trim line passed behind the malleolus, and the foot plate fitted the entire length of the plantar of the foot. The sole and calf shells had several holes for comfort, ease of ventilation, weight reduction, and material cost savings. The $3 \mathrm{D}$ printed insole used by Mannisi et al. (14) was produced by adding lateral wedges of $0^{\circ}, 5^{\circ}$, and $10^{\circ}$ to the insoles manufactured by $3 \mathrm{D}$ scanning the knees and ankles at a $90^{\circ}$-bend with no load applied. Tarrade et al. (15) scanned each patient's foot through a $3 \mathrm{D}$ footprint scanner and made customized orthoses. Subsequently, neutral insole was attached to the foot orthosis and applied to the subjects. Foot orthoses had an arch shape to support the arch.

\section{Spinal orthosis}

One prospective observational study was conducted to evaluate the effectiveness of 3D printed spinal orthosis (13).

The 3D printed orthosis used by Kuo et al. (13) was applied to the cervical vertebrae. Typical cervical orthosis wraps around the entire neck, whereas the $3 \mathrm{D}$ printed orthosis used in that study had support only in the front side. There were mandibular and lateral supports that descended to the clavicle at both ends of the mandibular support. In addition, the $3 \mathrm{D}$ printed orthosis had venting holes and strap holes on the mandibular support. The user could be fixed using the strap to prevent the orthosis from falling off the body.

\section{Discussion}

In the current review, we found that $3 \mathrm{D}$ printed orthoses can effectively improve biomechanical and kinematic parameters, and their effectiveness is similar to that of conventional orthoses. Additionally, study participants felt comfortable wearing 3D printed orthoses. Furthermore, in some studies, the effectiveness of 3D printed orthoses and the comfort when wearing them were better than those of conventional orthoses. Of the 22 studies included in our review, 10 of them $(3-5,7-11,13,19)$ compared the effectiveness of $3 \mathrm{D}$ printed orthoses with that of conventionally produced orthoses. In all those studies, 3D printed orthoses showed superior or similar results in the evaluation parameters (satisfaction, wrist spasticity, wristhand function, gait parameter, arch height index, and plantar foot pressure and joint range of motion (ROM) compared to conventional orthoses, and patient satisfaction with $3 \mathrm{D}$ printed orthoses was also comparable with that of conventional orthoses. The reasons why $3 \mathrm{D}$ printed orthoses were more satisfactory and some explanations are as follows:

(I) The surface of the body in contact with the orthosis is optimized in $3 \mathrm{D}$-printed orthoses.

Most conventional orthoses are made manually. Even if the orthosis is produced based on the patient's body casting, human error should not be overlooked. In particular, a slight difference in force can affect the metal orthoses when bending the stirrup or upright. In plastic orthosis, while making the negative plaster model, the plaster cloth wraps around the patient's body, which may create parts where the flesh is pressed and uneven. In addition, the conventional orthoses are worn by the patient after manufacture, and the operations of finely adjusting the angle, alignment, or circumference can be performed again. In contrast, the $3 \mathrm{D}$ printed orthoses are designed using computer programming and produced through a $3 \mathrm{D}$ printer. If the producer has accurate body dimensional information, there is a high probability that the output will be highly accurate.

(II) More solid support of patients' bodies than conventional orthoses, because the materials (polyurethane, light-activated resin, etc.) used in $3 \mathrm{D}$ printed orthoses have higher strength, which leads to better effects on controlling the ROM of joints. 
Strength refers to the resistance until the material breaks down. The strength decreases as the number of repeated and continuous loads to the orthosis increases. Therefore, it is important to consider the strength of the material when manufacturing an orthosis. However, strength is not the only factor to consider. In the case of an ankle-foot orthosis, if users wear an orthosis during gait, the impact on the orthosis every time they walk can be a problem. Therefore, toughness and brittleness, which are the characteristics of impact resistance, should be considered. In addition, as the plastic orthosis for the purpose of correction is applied with a continuous load, the orthosis may deform over time. In this case, creep and relaxation, which are the characteristics related to strain over time, should be considered.

(III) Increased comfort owing to the good distribution of pressure.

The distribution of pressure is influenced by a number of variables, including the shape of the body, size of the orthosis, structure of the orthosis, and motion of the affected area. The bone protrusion part or affected area needs space to avoid contact with the orthosis. If the orthosis is small and an excessive pressure is applied, adjustment is required. In the case of a flat foot, the load to the arch can be distributed by applying an arch support to the insole. The medial/lateral wedge or pad has the same function. The pressure is directly related to the user's comfort; therefore, it is important to consider it when designing an orthosis.

(IV) Lighter weight than conventional orthoses.

The weight of the orthosis is not limited to the selection of main material. By using $3 \mathrm{D}$ printing technology, the weight can be reduced with great precision by making small holes on the narrow surface. Making holes in the orthosis reduces weight and provides ventilation. In addition, designing to omit bolts, nuts, metal rings, etc., or outputting parts to a $3 \mathrm{D}$ printer can help reduce the weight of the orthosis.

The effects and satisfaction of $3 \mathrm{D}$ printed orthosis were also evaluated in studies not mentioned previously $(6,12,14-18,20-24)$. The results of stability, pain, pressure, function of the affected area, and satisfaction were generally excellent (Table 1). In aggregate, the pain was reduced, joint ROM was improved, and satisfaction was high because it was light and comfortable to wear. Given the advantages of $3 \mathrm{D}$ printed orthoses, this technology is expected to be used more widely in clinical practice in the near future. However, 3D printed orthosis is not excellent in all aspects. When performing a 3D scan, difficulty may arise owing to the patient being in an incorrect posture or continuously moving. In this case, a posture correction device should be used or an expert should set the optimal posture. There are limitations of the $3 \mathrm{D}$ printer as well. It is difficult to manufacture an orthosis with an exceptionally large circumference or height. In addition, although the price range varies, a printer with excellent quality and high production speed is expensive; therefore, it may be difficult to obtain a machine without basic funds. The use of unwarranted, low-cost printer and materials requires caution as it can cause secondary damage to users. In addition, for $3 \mathrm{D}$ printing technology to be actively used in the manufacturing of orthoses, we expect that practitioners in related fields, such as clinicians, prosthetists, orthotists, and occupational therapists, will have to acquire CAD utilization skills and increase their understanding of $3 \mathrm{D}$ printing technology.

Among the 22 studies included in our review, in 15 of them $(3-8,12-15,18-20,22,23)$, the subjects' bodies were scanned using a $3 \mathrm{D}$ scanner and later a CAD program was used to handle the data and design the orthosis. In five studies (9-11,17,21), a CAD program was directly used to design the orthosis, which was then self-produced. CAD files obtained through a 3D scanner can be modified using a CAD program, which is a design program that is used to create and edit drawings. Because CAD files can be stored for a long time, it is easy to manufacture an orthosis of the same design using a saved file when the orthosis is damaged or lost $(3,11)$. It is also easy to modify it according to the patient's desired shape $(9,19)$. Open-source design is a free design source. It is free to use and accessible by anyone, and a wider variety of designs can be created in a short period of time based on open-source data (21).

The materials usually used for $3 \mathrm{D}$ printed orthoses include PLA, ABS, and TPU. PLA is an environmentally friendly material that has no environmental hormones or heavy metals. Additionally, PLA is a material with excellent renewability and biocompatibility (25). ABS is a type of styrene resin composed of three components (acrylonitrile, butadiene, and styrene). ABS has high impact resistance and ease of processability (26). TPU has mechanical properties, such as the highest tensile strength, tearing strength, and abrasion resistance among thermoplastic elastomers (26). 
Therefore, these materials are being widely used when manufacturing objects with high durability.

In this review, we investigated the effects of $3 \mathrm{D}$ printed orthoses. In all reviewed studies, 3D printed orthoses were durable and showed similar or superior results in biomechanical parameters, kinematic parameters, and comfort evaluation compared to conventional orthoses. Based on these results, 3D printed orthoses appear to be of sufficient value to replace conventional orthoses in clinical practice. However, previous studies had a small percentage of randomized trials and fewer results comparing conventional and $3 \mathrm{D}$ printed orthoses. In particular, for a spinal orthosis, there is only one prospective observational study. In addition, some studies only confirmed satisfaction, which is an individual's subjective indicator. Therefore, for $3 \mathrm{D}$ printed orthoses to be more actively utilized in clinical practice, more randomized trials should be conducted to minimize the risk of bias and ensure the validity of the study. Moreover, it is necessary to compare the effects of conventional orthosis and $3 \mathrm{D}$ printed orthosis and evaluate objective indicators such as joint ROM, pressure distribution, and kinematic data.

\section{Acknowledgments}

Funding: The present study was supported by a National Research Foundation of Korea Grant funded by the Korean government (grant number NRF2019M3E5D1A02068106).

\section{Footnote}

Reporting Checklist: the authors have completed the NARRATIVE REVIEW reporting checklist. Available at http://dx.doi.org/10.21037/apm-20-1185

Conflicts of Interest: All authors have completed the ICMJE uniform disclosure form (available at http://dx.doi. org/10.21037/apm-20-1185). The authors have no conflicts of interest to declare.

Ethical Statement: The authors are accountable for all aspects of the work in ensuring that questions related to the accuracy or integrity of any part of the work are appropriately investigated and resolved.

Open Access Statement: This is an Open Access article distributed in accordance with the Creative Commons
Attribution-NonCommercial-NoDerivs 4.0 International License (CC BY-NC-ND 4.0), which permits the noncommercial replication and distribution of the article with the strict proviso that no changes or edits are made and the original work is properly cited (including links to both the formal publication through the relevant DOI and the license). See: https://creativecommons.org/licenses/by-nc-nd/4.0/.

\section{References}

1. Chae DS, Kim DH, Kang KY, et al. The functional effect of 3D-printing individualized orthosis for patients with peripheral nerve injuries: three case reports. Medicine (Baltimore) 2020;99:e19791.

2. Weinstein SL, Dolan LA, Wright JG, et al. Effects of bracing in adolescents with idiopathic scoliosis. $\mathrm{N} \mathrm{Engl} \mathrm{J}$ Med 2013;369:1512-21.

3. Cha YH, Lee KH, Ryu HJ, et al. Ankle-foot orthosis made by $3 \mathrm{~d}$ printing technique and automated design software. Appl Bionics Biomech 2017;2017:9610468.

4. Dombroski CE, Balsdon ME, Froats A. The use of a low cost $3 \mathrm{D}$ scanning and printing tool in the manufacture of custom-made foot orthoses: a preliminary study. BMC Res Notes 2014;7:443.

5. Jin H, Xu R, Wang S, et al. Use of 3D-printed heel support insoles based on arch lift improves foot pressure distribution in healthy people. Med Sci Monit 2019;25:7175-81.

6. Kim SJ, Kim SJ, Cha YH, et al. Effect of personalized wrist orthosis for wrist pain with three-dimensional scanning and printing technique: a preliminary, randomized, controlled, open-label study. Prosthet Orthot Int 2018;42:636-43.

7. Telfer S, Woodburn J, Collier A, et al. Virtually optimized insoles for offloading the diabetic foot: A randomized crossover study. J Biomech 2017;60:157-61.

8. Mo S, Leung SHS, Chan ZYS, et al. The biomechanical difference between running with traditional and 3D printed orthoses. J Sports Sci 2019;37:2191-7.

9. Xu R, Wang Z, Ma T, et al. Effect of 3D printing individualized ankle-foot orthosis on plantar biomechanics and pain in patients with plantar fasciitis: A randomized controlled trial. Med Sci Monit 2019;25:1392-400.

10. Xu R, Wang Z, Ren Z, et al. Comparative study of the effects of customized $3 \mathrm{~d}$ printed insole and prefabricated insole on plantar pressure and comfort in patients with symptomatic flatfoot. Med Sci Monit 2019;25:3510-9.

11. Zheng Y, Liu G, Yu L, et al. Effects of a 3D-printed 
orthosis compared to a low-temperature thermoplastic plate orthosis on wrist flexor spasticity in chronic hemiparetic stroke patients: a randomized controlled trial. Clin Rehabil 2020;34:194-204.

12. Allan R, Woodburn J, Telfer S, et al. Knee joint kinetics in response to multiple three-dimensional printed, customised foot orthoses for the treatment of medial compartment knee osteoarthritis. Proc Inst Mech Eng H 2017;231:487-98.

13. Kuo YR, Fang JJ, Wu CT, et al. Analysis of a customized cervical collar to improve neck posture during smartphone usage: a comparative study in healthy subjects. Eur Spine J 2019;28:1793-803.

14. Mannisi M, Dell'Isola A, Andersen MS, et al. Effect of lateral wedged insoles on the knee internal contact forces in medial knee osteoarthritis. Gait Posture 2019;68:443-8.

15. Tarrade T, Doucet F, Saint-Lô N, et al. Are custommade foot orthoses of any interest on the treatment of foot pain for prolonged standing workers? Appl Ergon 2019;80:130-5.

16. Chen YJ, Lin H, Zhang X, et al. Application of 3D-printed and patient-specific cast for the treatment of distal radius fractures: initial experience. 3D Print Med 2017;3:11.

17. Chu CH, Wang IJ, Sun JR, et al. Customized designs of short thumb orthoses using $3 \mathrm{D}$ hand parametric models. Assist Technol 2020. [Epub ahead of print].

18. Guida P, Casaburi A, Busiello T, et al. An alternative to plaster cast treatment in a pediatric trauma center using the CAD/CAM technology to manufacture customized threedimensional-printed orthoses in a totally hospital context: a feasibility study. J Pediatr Orthop B 2019;28:248-55.

Cite this article as: Choo YJ, Boudier-Revéret M, Chang MC. 3D printing technology applied to orthosis manufacturing: narrative review. Ann Palliat Med 2020;9(6):4262-4270. doi: 10.21037/apm-20-1185
19. Lee KH, Kim DK, Cha YH, et al. Personalized assistive device manufactured by $3 \mathrm{D}$ modelling and printing techniques. Disabil Rehabil Assist Technol 2019;14:526-31.

20. Liu Z, Zhang P, Yan M, et al. Additive manufacturing of specific ankle-foot orthoses for persons after stroke: a preliminary study based on gait analysis data. Math Biosci Eng 2019;16:8134-43.

21. Portnova AA, Mukherjee G, Peters KM, et al. Design of a 3D-printed, open-source wrist-driven orthosis for individuals with spinal cord injury. PLoS One 2018;13:e0193106.

22. Telfer S, Abbott M, Steultjens M, et al. Dose-response effects of customised foot orthoses on lower limb muscle activity and plantar pressures in pronated foot type. Gait Posture 2013;38:443-9.

23. Telfer S, Abbott M, Steultjens MP, et al. Dose-response effects of customised foot orthoses on lower limb kinematics and kinetics in pronated foot type. J Biomech 2013;46:1489-95.

24. Wang K, Shi Y, He W, et al. The research on 3D printing fingerboard and the initial application on cerebral stroke patient's hand spasm. BioMed Eng OnLine 2018;17:92.

25. Bai H, Deng S, Bai D, et al. Recent advances in processing of stereocomplex-type polylactide. Macromol Rapid Commun 2017. doi: http://dx.doi.org/10.1002/ marc. 201700454.

26. Memarian F, Fereidoon A, Ahangari MG. Effect of acrylonitrile butadiene styrene on the shape memory, mechanical, and thermal properties of thermoplastic polyurethane. J Vinyl Addit Techn 2018;24:E96-104. 